\title{
Numerical Study of Free Convection inside the Differentially Heated Cavity incorporating Variable Property $\mathrm{Al}_{2} \mathrm{O} 3-\mathrm{H}_{2} \mathrm{O}$ Nanofluid
}

DOI:10.36909/jer.13703

\author{
Bishwajit Sharma*, Md. Feroz Alam*, Mayur Krishna Bora*, Rabindra Nath Barman* \\ *Mechanical Engineering Department, National Institute of Technology Durgapur, West \\ Bengal, 713209, India \\ *Email: rn.barman@me.nitdgp.ac.in
}

\begin{abstract}
This paper investigates free convection in a partially heated square cavity filled with aluminawater nanofluid. The investigation is carried out at the three-volume fraction of nanoparticles $(0,0.03,0.05)$, two Prandtl numbers $(2.66,6)$, and constant Grashof number $\left(10^{5}\right)$ with three shapes of insulating obstacles (Square, Circular, and Rectangular). The results show that the nanofluid volume fraction and Prandtl number significantly enhance the heat transfer. The user-defined function (UDF) is developed and computed to investigate the effect of nanoparticle diameter and its temperature-dependent viscosity on convection. The average Nusselt number $(\mathrm{Nu})$ increased with the temperature-dependent viscosity model and by increasing the percentage concentration of the nanoparticles. For all obstacle shapes, the thermal performance improved with increase in the nano-particle diameter.
\end{abstract}

Keywords: Variable Property, Nanofluid, Differentially heated cavity, Fluent, UDF. 


\section{INTRODUCTION}

Fluids are the most practical medium for storing and transferring heat, and their thermal conductivity plays a significant role in energy transfer. Fluids like oil, water, and others have lower thermal conductivity. Therefore, a fluid with improved thermal conductivity is desired. Once compared to the base fluid, nanofluid delivers an efficient outcome with a considerable increase in heat transfer. A nanofluid is a homogeneous mixture of nanoparticles with required characteristics that are mixed with a base fluid. The addition of nanoparticles improves the thermal conductivity of the base fluid, improving its heat transport capacity. Nanofluids are used in heat exchangers for convection and microelectronics, ventilation, and micro-electro-mechanical systems. Choi et al., 1995, first concluded that nanofluid exhibits higher thermal conductivity than common base fluids. Godson et al., 2010, presented an overview by going through recent advances in mixed convection heat transfer using nanofluid. Their investigation summarized the enhancement of the fluid's thermal conductivity, and the increase in fluctuation due to the particles' chaotic movement enhances the heat transfer. Different researchers experimented with a wide range of experimental research in this field. Different researchers experimented with a wide range of experimental research in this field. Eastman et al., 1996, investigated the $5 \%$ volume fraction of $\mathrm{Al}_{2} \mathrm{O}_{3}$ and $\mathrm{CuO}$ water nanofluid and showed that the thermal conductivity enhanced with $\mathrm{CuO}$ at the same volume fraction. Xie et al., 2002, observed thermal conductivity enhancement by $30 \%$ when a $5 \%$ volume fraction of $\mathrm{Al}_{2} \mathrm{O}_{3}$ was mixed with ethylene glycol. It can be seen that researchers are experimenting with different nanofluids for a more improved and efficient energy exchanging process. The viscosity of a fluid is a property that is greatly influenced by its temperature. The experimental results by Lee et al., 1999, revealed that fluids containing oxide nanoparticles have much higher thermal conductivity compared to the fluid without oxide nanoparticles. They also analyzed that nanoparticle shape and size are also essential factors in increasing thermal conductivity. They showed that suspension of $13 \mathrm{~mm}$ mean diameter $\mathrm{Al}_{2} \mathrm{O}_{3}$ 
nanoparticles at $4.3 \%$ volume fraction in water increases thermal conductivity by $30 \%$. The $\mathrm{Al}_{2} \mathrm{O}_{3}-\mathrm{H}_{2} \mathrm{O}$ nanofluid in a differentially heated cavity examined by Hwang et al., 2007, showed the significant role of the nanoparticle's size and the average temperature in heat transfer inside the cavity, and $\mathrm{Al}_{2} \mathrm{O}_{3}-\mathrm{H}_{2} \mathrm{O}$ nanofluid was found to be more stable than the base fluid. Ho et al. proposed different models for change in thermal conductivity and dynamic viscosity of the Alumina-water nanofluid and showed that other models have variable outcomes on the heat transfer process. Jang et al., 2004, showed that temperature distribution inside the cavity filled with nanofluid is highly affected by nanoparticle concentration, size, and heat exchange between hot and cold sides. The significance of the heater location within the enclosure was examined by Panigrahi et al., 2019. They found out the role of the heater location for obtaining improved heat transfer. The addition of nanoparticles to the base fluid enhanced total heat flow from the hot to cold regions inside the cavity. Heat transport becomes efficient when a more significant volume concentration of nanofluid is incorporated. A recent research work showed the effectiveness of nanofluids in different industrial processes likes channel flows (Sheikhzadeh et al., 2019 and Eiamsa-ard et al. 2018), heat exchangers (Du et al., 2020 and Zheng et al. 2020), and solar collectors (Hussein et al., 2020 and Tong et al., 2020).

Despite the enhanced thermal conductivity of the nanofluids, thermal mixing is an essential parameter for the heat transfer inside the enclosure. Several studies were carried out to enhance the thermal mixing by employing the obstacles inside the closed enclosures filled with various types of water-based nanofluids (Bora et al., 2020 and Sharma et al., 2021). The investigation of $\mathrm{Wu}$ et al., 2009, concludes that $\mathrm{Al}_{2} \mathrm{O}_{3}-\mathrm{H}_{2} \mathrm{O}$ nanofluid at higher concentrations tends to aggregate. Their study also deals with the enhancement of the freezing rate of nanofluid. The freezing time of $\mathrm{Al}_{2} \mathrm{O}_{3}-\mathrm{H}_{2} \mathrm{O}$ nanofluid is reduced by $20.5 \%$ on the addition of $0.2 \%$ by the weight of $\mathrm{Al}_{2} \mathrm{O}_{3}$ nanoparticles. Previously, many studies based on mixed convection in a shear-driven cavity using nanofluid and using a standard homogeneous flow method to examine them have been published. However, previous experimental and numerical 
studies showed that the current mathematical homogenous model underestimates the nanofluid heat transfer performance. The nanoparticles' size and shape are the most critical factors determining the nanofluid's diffusion and thermo-physical properties. Although the thermo-physical properties vary with the temperature change, very few works are available related to the mathematical modeling of these physical properties with varying temperatures. An effort is made in this manuscript to find out the effect of temperature and nanoparticle diameter on the thermo-physical properties by obtaining a suitable mathematical model to study the thermal behavior of the nanofluid inside the enclosure. The present work analyses the effects of nanoparticle diameter and temperature on nanofluid's thermo-physical properties, further defining the thermal performance at different parameters.

\section{MATHEMATICAL MODELLING}

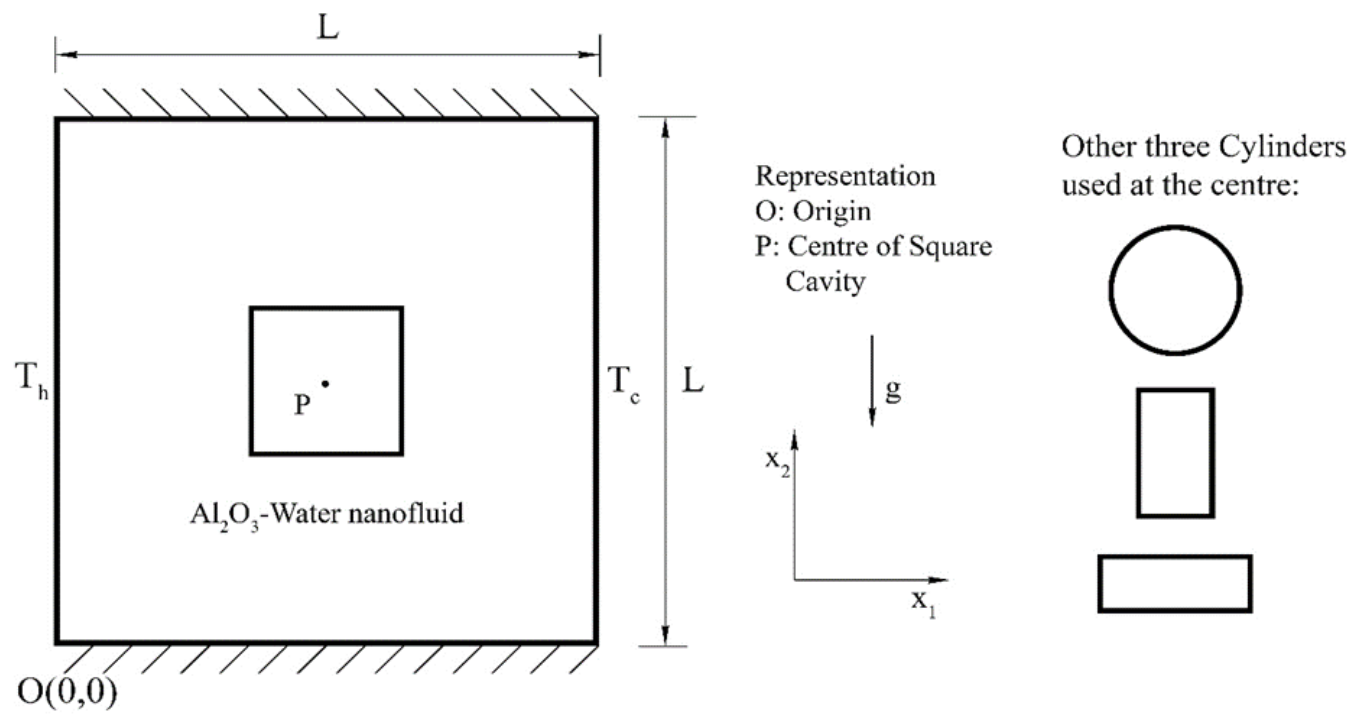

Figure 1.The schematic diagram of the domain with the boundary condition

The square enclosure is filled with $\mathrm{Al}_{2} \mathrm{O}_{3}-\mathrm{H}_{2} \mathrm{O}$ nanofluid with top and bottom walls maintained at the adiabatic condition. All the cavity walls are stationary, with the primary objective to understand the free convection inside them. The temperature gradient between the left vertical hot wall and the right vertical cold wall causes fluid and heat to circulate inside the cavity. Figure 1 illustrates the schematics of a square cavity obstacle of various shapes. Aspect ratio is one of the critical parameters that influence the flow dynamics inside the fluid domain with a blockage, and it is defined as the ratio of the characteristic length of the 
obstacle to the length of the square enclosure. Different shaped obstacles with varying aspect ratios are placed at the center of the enclosure. The origin and the center of the cavity are represented by $\mathrm{O}$ and $\mathrm{P}$, respectively. A No-slip boundary condition is imposed on the wall of the enclosure. The governing equations assume that all the thermo-physical properties of the fluid are constant with temperature except density and dynamic viscosity. The density is defined as per Boussinesq's approximation along with the temperature-dependent viscosity. The nanofluid is considered to be Newtonian and incompressible. The nanoparticle's shape is spherical, and the size is less than $100 \mathrm{~nm}$, so the continuum is valid.

\section{Governing equations}

The dimensional form of the equations involved in the investigation are

Continuity equation

$$
\frac{\partial u_{1}}{\partial x_{1}}+\frac{\partial u_{2}}{\partial x_{2}}=0
$$

$\mathrm{x}$-momentum equation:

$$
\frac{\partial u_{1}}{\partial t}+u_{1} \frac{\partial u_{1}}{\partial x_{1}}+u_{2} \frac{\partial u_{1}}{\partial x_{2}}=-\frac{1}{\rho_{n f}} \frac{\partial p}{\partial x_{1}}+\frac{\mu_{n f}}{\rho_{n f}}\left(\frac{\partial^{2} u_{1}}{\partial x_{1}^{2}}+\frac{\partial^{2} u_{1}}{\partial x_{2}^{2}}\right)
$$

y-momentum equation:

$$
\frac{\partial u_{2}}{\partial t}+u_{1} \frac{\partial u_{2}}{\partial x_{1}}+u_{2} \frac{\partial u_{2}}{\partial x_{2}}=-\frac{1}{\rho_{n f}} \frac{\partial p}{\partial x_{2}}+\frac{\mu_{n f}}{\rho_{n f}}\left(\frac{\partial^{2} u_{2}}{\partial x_{1}^{2}}+\frac{\partial^{2} u_{2}}{\partial x_{2}^{2}}\right)+\frac{1}{\rho_{n f}}\left[(1-\phi) \rho_{f} \beta_{f}+\phi \rho_{s} \beta_{s}\right] g\left(T-T_{L}\right)
$$

Energy equation:

$$
\frac{\partial T}{\partial t}+u_{1} \frac{\partial T}{\partial x_{1}}+u_{2} \frac{\partial T}{\partial x_{2}}=\alpha_{n f}\left(\frac{\partial^{2} T}{\partial x_{1}^{2}}+\frac{\partial^{2} T}{\partial x_{2}^{2}}\right)
$$

Here, $\alpha_{n f}=\frac{k_{n f}}{\left(\rho C_{p}\right)_{n f}}$

Equations in the non-dimensional form are written as

$$
\begin{aligned}
& \frac{\partial U_{1}}{\partial X_{1}}+\frac{\partial U_{2}}{\partial X_{2}}=0 \\
& \frac{\partial U_{1}}{\partial t^{*}}+U_{1} \frac{\partial U_{1}}{\partial X_{1}}+U_{2} \frac{\partial U_{1}}{\partial X_{2}}=-\frac{\rho_{f}}{\rho_{n f}} \frac{\partial P}{\partial X_{1}}+\frac{\rho_{f} \mu_{e f f} \operatorname{Pr}}{\rho_{n f} \mu_{f}}\left(\frac{\partial^{2} U_{1}}{\partial X_{1}^{2}}+\frac{\partial^{2} U_{1}}{\partial X_{2}^{2}}\right)
\end{aligned}
$$




$$
\begin{aligned}
& \frac{\partial U_{2}}{\partial t^{*}}+U_{1} \frac{\partial U_{2}}{\partial X_{1}}+U_{2} \frac{\partial U_{2}}{\partial X_{2}}=-\frac{\rho_{f}}{\rho_{n f}} \frac{\partial P}{\partial X_{2}}+\frac{\mu_{e f f} \rho_{f} \operatorname{Pr}}{\rho_{n f} \mu_{f}}\left(\frac{\partial^{2} U_{2}}{\partial X_{1}^{2}}+\frac{\partial^{2} U_{2}}{\partial X_{2}^{2}}\right)+\frac{(1-\phi) \rho_{f} \beta_{f}+\phi \rho_{s} \beta_{s}}{\rho_{n f} \beta_{f}} \operatorname{Pr}^{2} \operatorname{Gr} \theta \\
& \frac{\partial \theta}{\partial t^{*}}+U_{1} \frac{\partial \theta}{\partial X_{1}}+U_{2} \frac{\partial \theta}{\partial X_{2}}=\frac{\alpha_{n f}}{\alpha_{f}}\left(\frac{\partial^{2} \theta}{\partial X_{1}^{2}}+\frac{\partial^{2} \theta}{\partial X_{2}^{2}}\right)
\end{aligned}
$$

2.2 Various dimensionless parameters used above are formulated as follows:

\begin{tabular}{|ll|l|l|l|}
\hline$X_{1}=\frac{x_{1}}{L}$ & (9) & $X_{2}=\frac{x_{2}}{L}$ & (10) & $U_{1}=\frac{u_{1}}{\alpha_{f} / L}$ \\
\hline$U_{2}=\frac{u_{2}}{\alpha_{f} / L}$ & $(12)$ & $t^{*}=\frac{\alpha_{f} t}{L^{2}}$ & (13) & $\theta=\frac{\left(T-T_{c}\right)}{\left(T_{h}-T_{c}\right)}$ \\
\hline$P=\frac{p}{\rho_{f}\left(\alpha_{f} / L\right)^{2}}$ & $(15)$ & $\operatorname{Pr}=\frac{\left(\mu C_{p}\right)_{n f}}{K_{n f}}$ & (16) & $G r=\frac{\rho_{f}^{2} g \beta\left(T_{h}-T_{c}\right) L^{3}}{\mu_{f}^{2}}$
\end{tabular}

\section{Effective properties of the nanofluid}

\section{Density}

$$
\rho_{n f}=(1-\phi) \rho_{f}+\phi \rho_{s}
$$

2. Heat capacitance

$$
\left(C_{p}\right)_{n f}=\left[(1-\phi)\left(\rho C_{p}\right)_{f}+\phi\left(\rho C_{p}\right)_{s}\right] / \rho
$$

\section{Thermal conductivity}

Stagnant thermal conductivity as proposed by Maxwell-Garnett's approximation model:

$$
\frac{k_{\text {stationary }}}{k_{f}}=\frac{k_{s}+2 k_{f}-2 \phi\left(k_{f}-k_{s}\right)}{k_{s}+2 k_{f}+\phi\left(k_{f}-k_{s}\right)}
$$

The nanoparticle is considered spherical in shape. In this paper, conductivity is calculated by considering the size of the nanoparticle as $5 \mathrm{~nm}$ in diameter. The requisite formula is given by $\mathrm{Xu}$ et al. (2006). In this formula value of c (an empirical constant) for water is taken as 85 .

$$
\frac{k_{n f}}{k_{f}}=\frac{k_{\text {stationary }}}{k_{f}}+\frac{k_{c}}{k_{f}}=\frac{k_{s}+2 k_{f}-2 \phi\left(k_{f}-k_{s}\right)}{k_{s}+2 k_{f}+\phi\left(k_{f}-k_{s}\right)}+c \frac{N u_{p} d_{f}}{\operatorname{Pr}} \frac{\left(2-D_{f}\right) D_{f}}{\left(1-D_{f}\right)^{2}} \frac{\left[\left(\frac{d_{p, \max }}{d_{p, \text { min }}}\right)^{1-D_{f}}-1\right]^{2}}{\left(\frac{d_{p, \max }}{d_{p, \text { min }}}\right)^{2-D_{f}}-1} \frac{1}{d_{p}}
$$

Where $\mathrm{D}_{\mathrm{f}}$ is known as fractal dimension and $\mathrm{d}_{\mathrm{p}}$ is nanoparticle mean diameter, and $\mathrm{Nu}_{\mathrm{p}}$ is 2 . The $\mathrm{D}_{\mathrm{f}}, \mathrm{d}_{\mathrm{p}, \min }, \mathrm{d}_{\mathrm{p}, \max }$ are given by 


$$
\begin{aligned}
& D_{f}=2-\frac{\ln \phi}{\ln \left(d_{p, \text { min}} / d_{p, \text { max }}\right)} \\
& d_{p, \text { min }}=d_{p} \cdot \frac{D_{f}-1}{D_{f}} \\
& d_{p, \text { max }}=d_{p} \frac{D_{f}-1}{D_{f}}\left(\frac{d_{p, \text { min }}}{d_{p, \text { max }}}\right)^{-1}
\end{aligned}
$$

4. Dynamic viscosity

$$
\mu_{H_{2} \mathrm{O}}=\left(1.2723 T_{r c}^{5}-8.736 T_{r c}^{4}+33.708 T_{r c}^{3}-246.6 T_{r c}^{2}+518.78 T_{r c}+1153.9\right) \times 10^{-6}
$$

Where, $T_{r c}=\log (T-273)$

$$
\mu_{n f}=\mu_{H_{2} O}(1+2.5 \phi)\left(1+\eta\left(\frac{d_{p}}{L}\right)^{-2 \varepsilon} \phi^{\frac{2}{3}}(\varepsilon+1)\right)
$$

Here $\varepsilon$ and $\eta$ empirical constant taken as 0.25 and 280 respectively [13]

\section{Boundary conditions in non-dimensional form:}

$$
\begin{aligned}
& u_{1}=u_{2}=\frac{\partial \theta}{\partial X_{2}}=0 \text { at } X_{2}=0 \text { for } 0<X_{1}<1 \\
& u_{1}=u_{2}=\frac{\partial \theta}{\partial X_{2}}=0 \text { at } X_{2}=1 \text { for } 0<X_{1}<1 \\
& u_{1}=u_{2}=0 ; \theta=0 \text { at } X_{1}=1 \text { for } 0 \leq X_{2} \leq 1 \\
& u_{1}=u_{2}=0 ; \theta=1 \text { at } X_{1}=0 \text { for } 0 \leq X_{2} \leq 1
\end{aligned}
$$

\section{Nusselt number}

The local Nusselt number is defined at the cold wall as

$$
N u=-\frac{k_{n f}}{k_{f}}\left(\frac{\partial \theta}{\partial X_{1}}\right)
$$

Similarly the average Nusselt number is

$$
N u_{\text {avg }}=\int_{0}^{1} \frac{N u}{X_{2}} \cdot d X_{2}
$$

Table 1. Thermo-physical properties of the materials used in the study at $300 \mathrm{~K}$

\begin{tabular}{|l|l|l|l|l|}
\hline & $\rho\left(\mathrm{kg} / \mathrm{m}^{3}\right)$ & $\mathrm{C}_{\mathrm{p}}(\mathrm{J} / \mathrm{kg} \mathrm{K})$ & $\mathrm{k}(\mathrm{W} / \mathrm{m} \mathrm{K})$ & $\beta(1 / \mathrm{K})$ \\
\hline
\end{tabular}




\begin{tabular}{|l|l|l|l|l|}
\hline Pure water & 998.2 & 4182 & 0.6 & $2.1 \times 10^{-4}$ \\
\hline $\mathrm{Al}_{2} \mathrm{O}_{3}$ & 3900 & 850 & 46 & $1.67 \times 10^{-5}$ \\
\hline Aluminium & 2719 & 871 & 202.4 & - \\
\hline
\end{tabular}

\section{NUMERICAL SOLUTION}

The continuity, momentum, and energy equations are computed using the finite volume method. The pressure-velocity coupling is solved using the SIMPLE scheme, while energy and momentum are solved using the second-order upwind and QUICK schemes, respectively. The convergence criteria for energy is taken to be the order of $10^{-12}$, whereas, for continuity and momentum, it is in order of $10^{-8}$ and $10^{-9}$, respectively.

\section{Grid Independence Test}

At each grid count, the grid independence test is conducted, and $\mathrm{Nu}_{\text {avg. }}$ is determined. The observation is made for $\mathrm{Gr}=10^{5}$ by considering six different mesh configurations $(31 * 31$, $61 * 61,81 * 81,101 * 101,121 * 121$, and $161 * 161)$, and accordingly, $\mathrm{Nu}_{\mathrm{avg}}$ is calculated. $\mathrm{Nu}_{\mathrm{avg}}$ deviation is found to be lesser than 0.0025 percent for grid counts greater than $121 * 121$.

\section{Numerical Model Validation}

The work of Lin et al., 2014, at $\mathrm{Gr}=10^{5}, \phi=0.05, \mathrm{~d}_{\mathrm{p}}=5 \mathrm{~nm}$, and $\operatorname{Pr}=6$ is used to compare the present numerical scheme, and a good agreement is found and is shown in Table 2.

Table2. The average Nusselt number comparison of effect of different diameter of nanoparticles with the past work

\begin{tabular}{|c|c|c|c|}
\hline $\begin{array}{c}\mathrm{R} \text { (ratio of minimum diameter of the } \\
\text { nanoparticle to maximum diameter) }\end{array}$ & $\begin{array}{c}\text { Lin et al., 2014 } \\
\mathrm{Nu}_{\mathrm{avg}}\end{array}$ & Present study & Error \% \\
\hline 0.001 & 9.45 & 9.53 & 0.84 \\
\hline 0.004 & 10.21 & 10.10 & 1.07 \\
\hline 0.005 & 10.71 & 10.5 & 1.9 \\
\hline
\end{tabular}




\section{RESULTS AND DISCUSSIONS}

In this paper, heat convection inside a square cavity filled with temperature-dependent viscosity of nanofluid is studied. The buoyancy-driven flow inside the differentially heated cavity is considered at $\mathrm{Gr}=10^{5}$ for $\operatorname{Pr}=2.66$ and $\operatorname{Pr}=6$. Streamline and isotherms of three different volume fractions $(\phi=0,0.03,0.05)$ of nanoparticles and four different shapes of insulating obstacles (square, circle, horizontal rectangle, and vertical rectangle are compared in order to comprehend the flow and thermal behaviour inside the cavity. Figure 2 represents the streamlines for different shaped obstacles at $\operatorname{Pr} 2.66$ and 6. From the streamlines, it can be noted that the size of the eddy present on the right side of the obstacle goes on increasing with increasing $\phi$. As a result, the area of fluid flowing between the eddy and the cavity's right wall is reduced, leading to an improved heat transfer at the cold wall region. From Isotherms at $\operatorname{Pr}=6$, as shown in figure 4, the high-temperature isotherms, e.g., $0.78 \& 0.72$, are closer to the cold wall with an increase in $\phi$, resulting in an enhanced heat transfer. At $\operatorname{Pr}=2.66$, for 0.05 volume fraction, maximum $\mathrm{Nu}$ is obtained in case of a circular obstacle. It is due to uniformity in the flow field surrounding the circular obstacle.
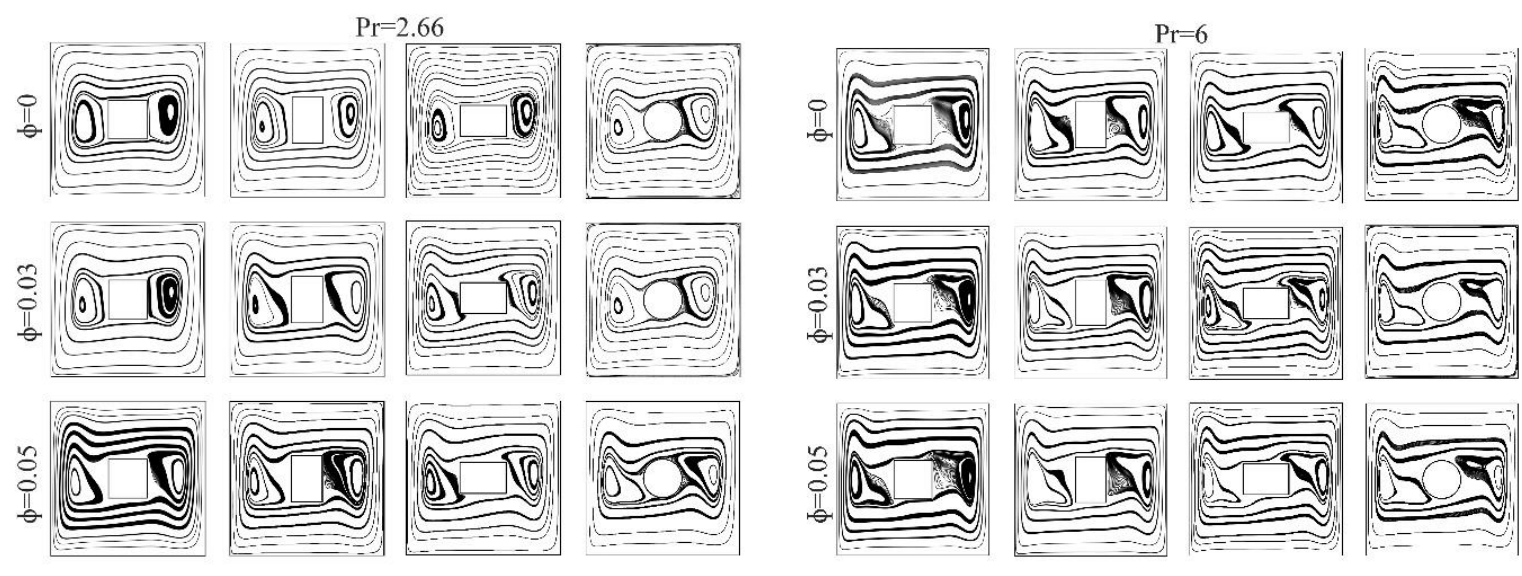

Figure 2. Streamlines for a different shaped obstacle at the two Pr number

Figure 3 shows the isotherms at three different volume concentrations of nanofluid. Isotherms at $\operatorname{Pr}=6$ are observed closer to the cold wall than $\operatorname{Pr}=2.66$. At higher $\operatorname{Pr}$, this causes an increase in the average Nusselt number $\left(\mathrm{Nu}_{\mathrm{avg}}\right)$ at the cold wall. Here, Viscosity changes with temperature and average diameter of the nanoparticle. Its effect can be seen directly from the 
calculation of $\mathrm{Nu}_{\text {avg }}$ at the cold wall. A significant drop in the viscosity occurs as the temperature rises, resulting in an increase in $\mathrm{Nu}_{\text {avg. }}$. This is in contrast to the cases where viscosity remains constant.
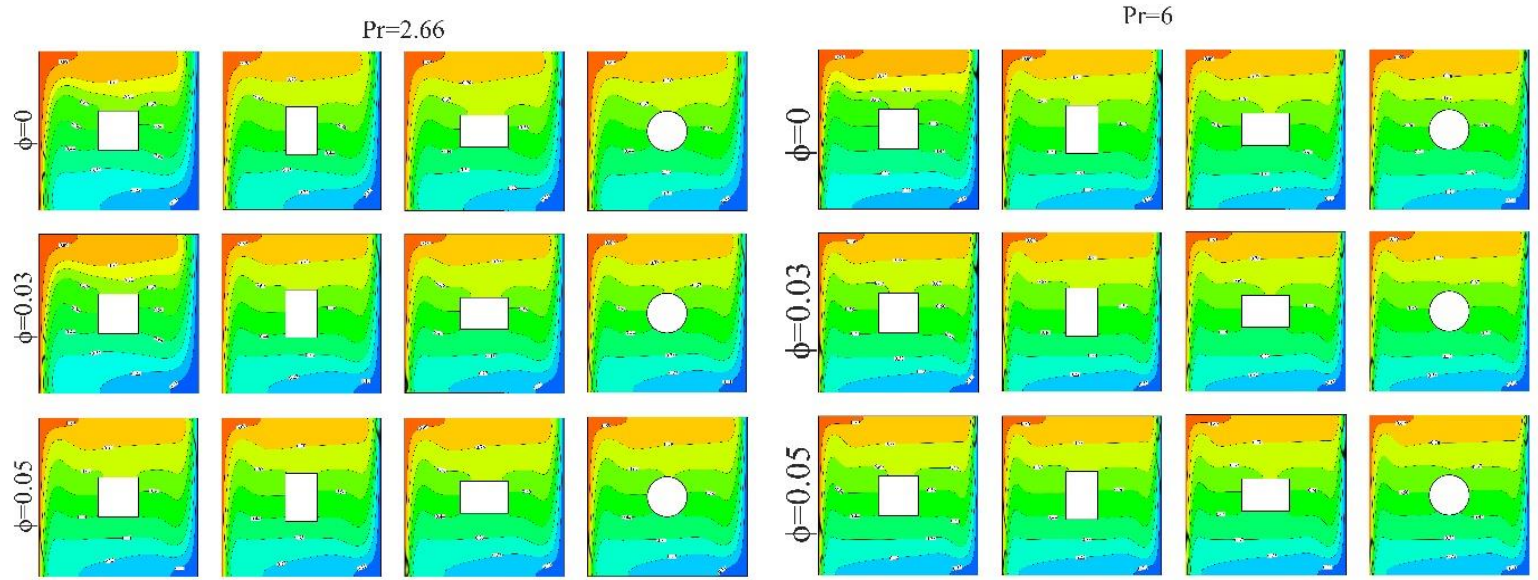

Figure 3. Isotherms for a different shaped obstacle at two Prandtl number
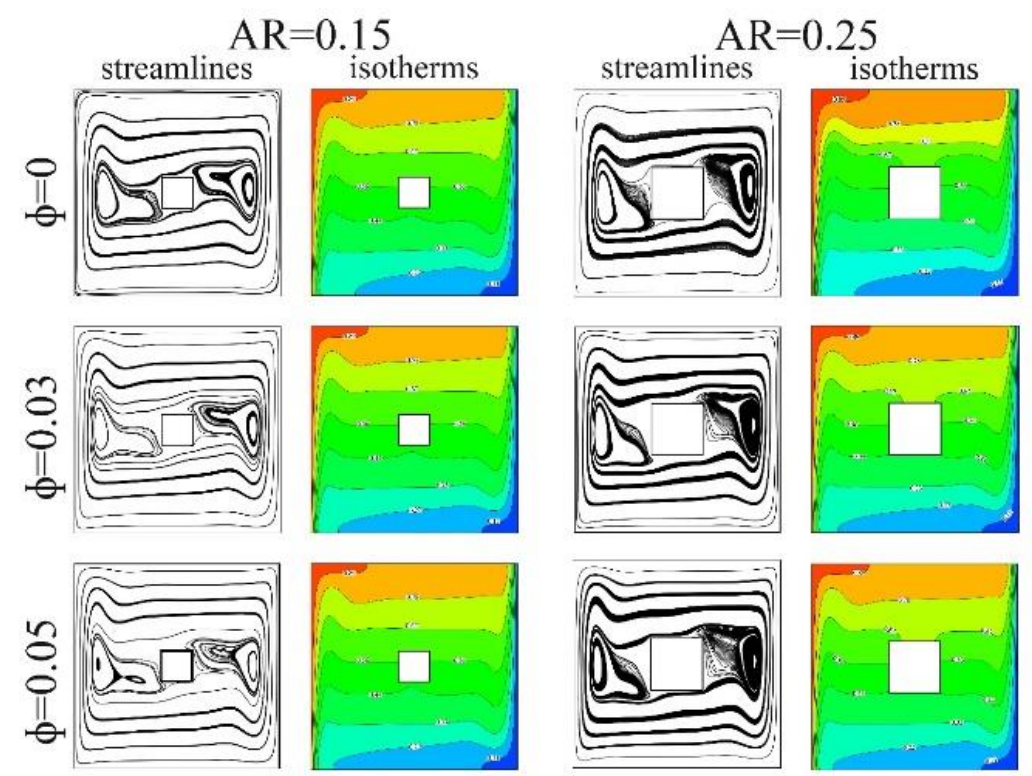

Figure 4. Streamlines and isotherms for a square-shaped obstacle at two aspect ratio. 

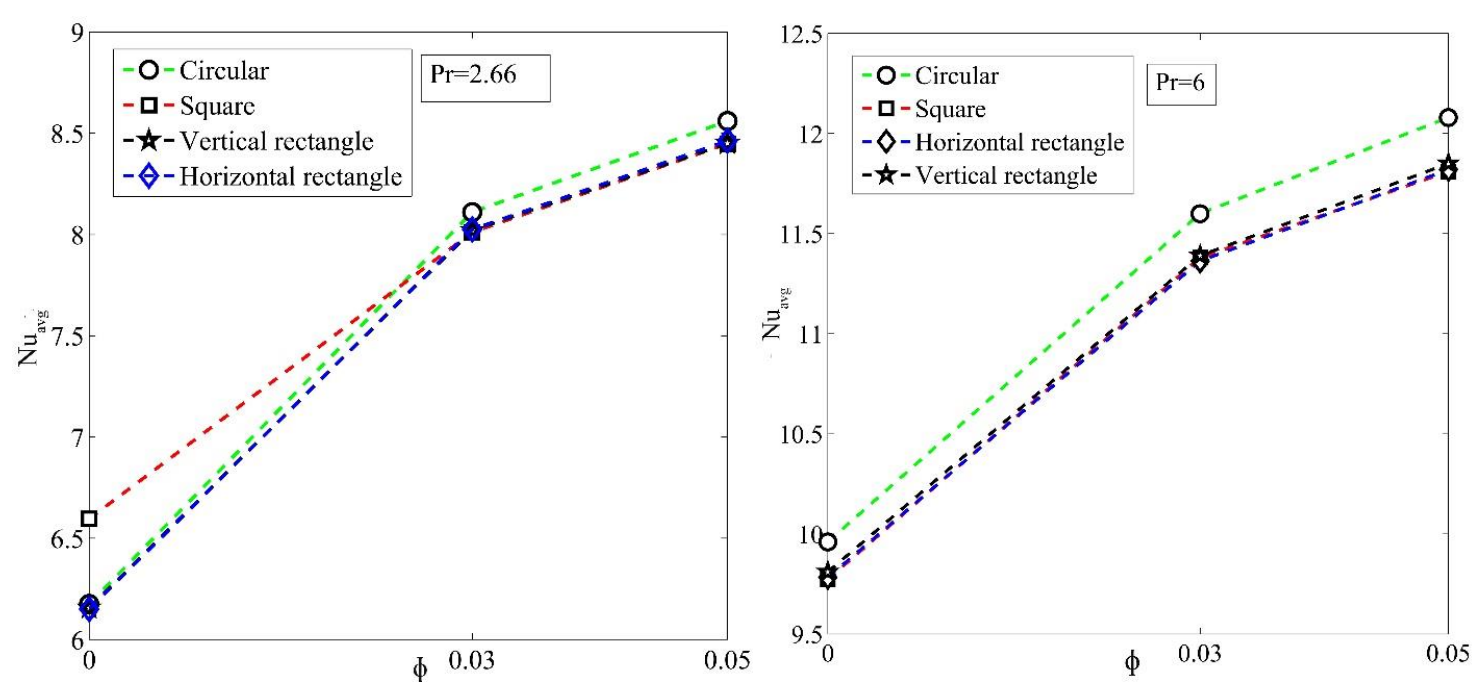

Figure 5. Variation of average Nusselt number at the two Pr.

The streamlines and isotherms for the cavity enclosing square-shaped obstacle are shown in figure 4 at $5 \%$ volume fraction of two different aspect ratios. It is observed that the aspect ratio of the obstacles plays an essential role in enhancing the heat transfer between the cavity walls along with the thermal mixing. An increase in aspect ratio results in a rise in $\mathrm{Nu}_{\text {avg }}$ at the cold wall. This is because of the presence of restrictions in the flow field. Due to this, flow velocity increases, and more amount of heat reaches the cold wall. Isotherms primarily heat up the upper portion of the cold wall at both Pr. This means that maximum heat is carried to the upper part of the cold wall compared to the bottom part. Hence, this could be one possible case of study in which one has to heat the cold wall more on one side than the other side.

A minimal change in the flow and thermal fields is observed for the rectangular obstacle at both orientations. Hence, no significant difference in the $\mathrm{Nu}_{\mathrm{avg}}$ is seen between both cases. At $\operatorname{Pr}=6$, maximum heat transfer occurs for circular obstacles at all the volume fractions of nanofluid. The uniformity of flow around the circular obstacle contributes to this enhancement. Also, some of the isotherms, e.g., 0.67 line, are not reaching the obstacle for all cases of the circular obstacle. This implies that whatever heat is carried by that line directly goes to the cold wall. However, different patterns are seen in other shapes where isotherms initially heat the insulated obstacle then transfer heat to the cold wall. As a result, a portion of the heat flux is wasted, resulting in an overall decrease in $\mathrm{Nu}_{\mathrm{avg}}$ at the cold wall.

Nusselt number is the critical parameter for determining the amount of heat transfer in any 
thermal domain. The $\mathrm{Nu}_{\text {avg }}$ at the cold wall for both Prandtl numbers is shown in Figure 5. It can be seen that $\mathrm{Nu}_{\text {avg }}$ increases with an increase in $\phi$ and Pr. Figure 6 represents the effect of change of viscosity with temperature on $\mathrm{Nu}$. With user-defined function (UDF), where viscosity is changing with temperature Nu increases considerably.

Figure 7 shows the variation in $\mathrm{Nu}_{\text {avg }}$ for different diameters of the nanoparticle at $\operatorname{Pr} 2.66$. With the increasing size of the particles, the heat transfer reduces. The same type of pattern for heat reduction is observed at Pr 6 for different-shaped obstacles. At a specific size of the nano-particle, an increase in $\mathrm{Pr}$ results in an increase in $\mathrm{Nu}_{\text {avg. }}$.
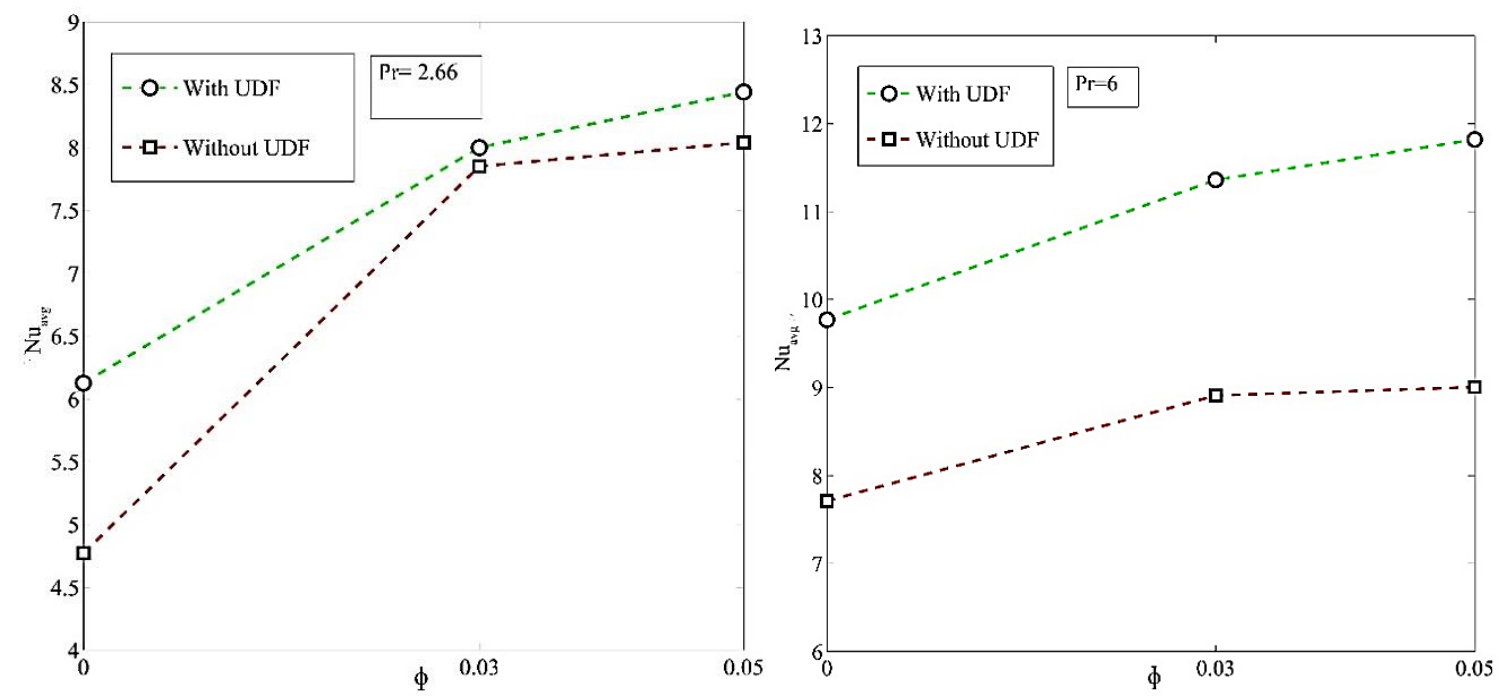

Figure 6. $\mathrm{Nu}_{\text {avg }}$ variation for square obstacle with and without UDF for viscosity.
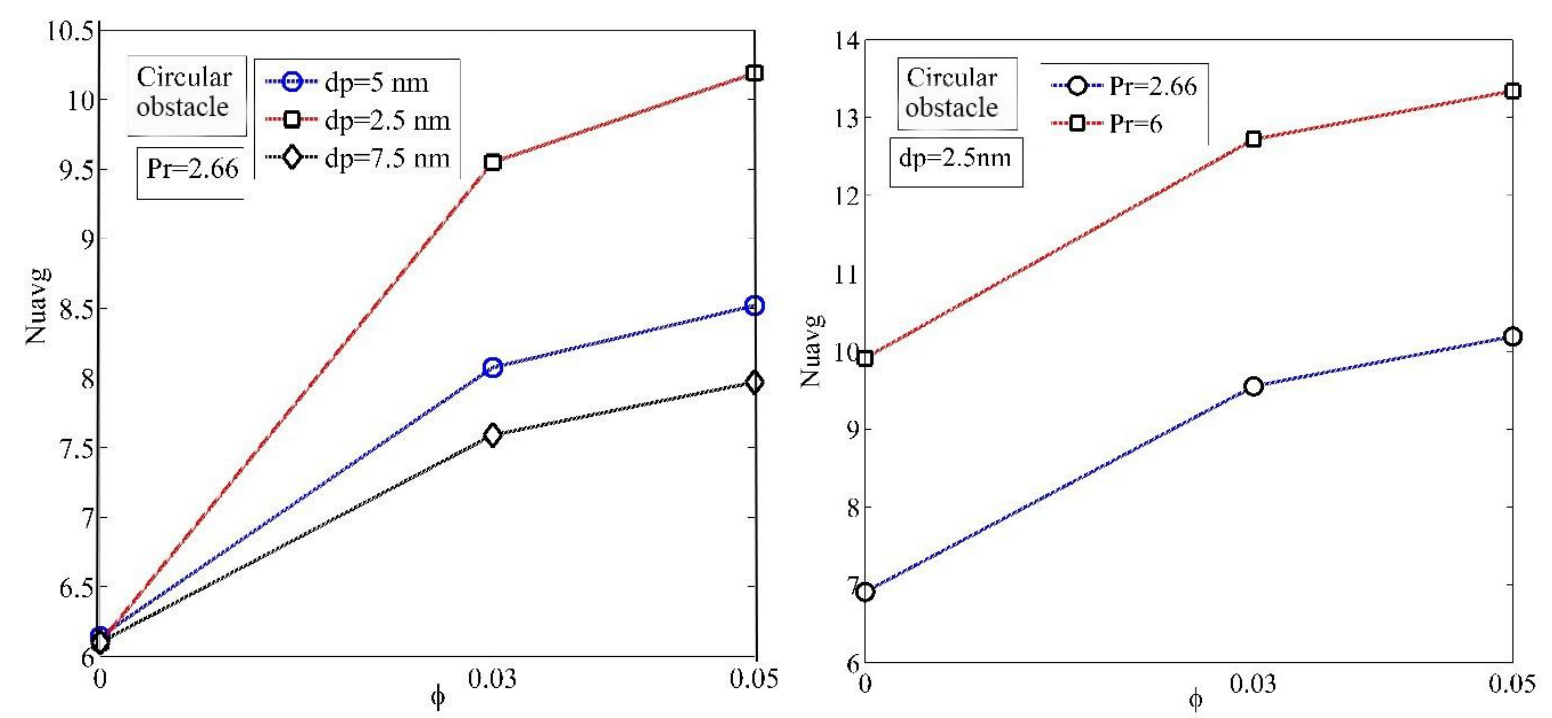

Figure 7. $\mathrm{Nu}_{\mathrm{avg}}$ variation for (a) different $\mathrm{d}_{\mathrm{p}}$, and, (b) different $\mathrm{Pr}$ 


\section{CONCLUSIONS}

The study illustrates the effect of viscosity variation with temperature on the flow and thermal field. The numerical investigation is carried out in the buoyancy-driven flow inside a cavity with different shapes of the obstacle with the varying volume fraction of nanofluids, resulting in an increase in Nusselt number with an increase in $\phi$ and Pr. Among all the obstacle shapes, the circular obstacle enhanced heat transfer significantly at a specific Prandtl number. As the nanoparticle diameter increases, the average $\mathrm{Nu}$ increases with an increase in $\phi$ for each obstacle. An increase in the aspect ratio also increased the heat transfer due to improved thermal mixing.

\section{REFERENCES}

Bora, M. K., Alam, M. F., Sharma, B., \& Barman, R. N. 2019. Numerical investigation of Ag-H2O nanofluid in a lid driven square cavity with different shaped conducting and insulating cylinders placed at centre. International Journal of Heat and Technology, Vol. 37, No. 3, pp. $831-838$

Choi, S. U., \& Eastman, J. A. 1995. Enhancing thermal conductivity of fluids with nanoparticles (No. ANL/MSD/CP-84938; CONF-951135-29). Argonne National Lab., IL (United States).

Du, R., Jiang, D., Wang, Y. \& Shah, K.W., 2020. An experimental investigation of $\mathrm{CuO}$ /water nanofluid heat transfer in geothermal heat exchanger. Energy and Buildings, 227, p.110402.

Eastman, J., Choi, U., Li, S., Thompson, L., \& Lee, S. 1996. Enhanced Thermal Conductivity through the Development of Nanofluids. MRS Proceedings, 457, 3.

Godson, L., Raja, B., Lal, D. M., \& Wongwises, S. E. A. 2010. Enhancement of heat transfer using nanofluids - an overview. Renewable and sustainable energy reviews, $14(2), 629-641$.

Ho, C. J., Chen, M. W., \& Li, Z. W. 2008. Numerical simulation of natural convection 
of nanofluid in a square enclosure: effects due to uncertainties of viscosity and thermal conductivity. International Journal of Heat and Mass Transfer, 51(17-18), 4506-4516.

Hussein, O.A., Habib, K., Muhsan, A.S., Saidur, R., Alawi, O.A. \& Ibrahim, T.K., 2020. Thermal performance enhancement of a flat plate solar collector using hybrid nanofluid. Solar Energy, 204, pp.208-222.

Hwang, K. S., Lee, J. H., \& Jang, S. P. 2007. Buoyancy-driven heat transfer of waterbased $\mathrm{Al}_{2} \mathrm{O}_{3}$ nano fluids in a rectangular cavity. International Journal of Heat and Mass Transfer, 50(19-20), 4003-4010.

Jang, S.P. \& Choi, S.U., 2004. January. Free convection in a rectangular cavity (Benard convection) with nanofluids. ASME International Mechanical Engineering Congress and Exposition (Vol. 4711, pp. 147-153).

Khoshvaght-Aliabadi, M., Hormozi, F. \& Zamzamian, A., 2014. Effects of geometrical parameters on performance of plate-fin heat exchanger: Vortex-generator as core surface and nanofluid as working media. Applied Thermal Engineering, 70(1), pp.565-579.

Li, S., \& Eastman, J. A. 1999. Measuring thermal conductivity of fluids containing oxide nanoparticles. J. Heat Transf, 121(2), 280-289.

Lin, K.C. \& Violi, A., 2010. Natural convection heat transfer of nanofluids in a vertical cavity: Effects of non-uniform particle diameter and temperature on thermal conductivity. International Journal of Heat and Fluid Flow, 31(2), pp.236-245. 5.

Panigrahi, A., Sharma, B., \& Barman, R. N. 2019. Numerical investigation of mixed convection incorporating $\mathrm{Ag}-\mathrm{H}_{2} \mathrm{O}$ nano fluid inside square enclosure for different heater locations. International Journal of Mathematical, Engineering and Management Sciences, 4(2): 442-451.

Sharma B., Bora M. K., Alam, M. F., \&Barman R. N., 2021. Mixed Convection Inside a Cavity Incorporating $\mathrm{Cu}-\mathrm{H}_{2} \mathrm{O}$ Nanofluid with Conducting Cylinders Placed at Optimum Position. J. Nano- Electron. Phys. 13 No 3, 03008

Sheikhzadeh, G.A., Barzoki, F.N., Arani, A.A.A. \& Pourfattah, F., 2019. Wings shape 
effect on behavior of hybrid nanofluid inside a channel having vortex generator. Heat and Mass Transfer, 55(7), pp.1969-1983.

Tong, Y., Chi, X., Kang, W. \& Cho, H., 2020. Comparative investigation of efficiency sensitivity in a flat plate solar collector according to nanofluids. Applied Thermal Engineering, 174, p.115346.

Wu, S., Zhu, D., Li, X., Li, H., \& Lei, J. 2009. Thermal energy storage behavior of $\mathrm{Al}_{2} \mathrm{O}_{3}-\mathrm{H}_{2} \mathrm{O}$ nanofluids. Thermochimica Acta, 483(1-2), 73-77.

Xie, H., Wang, J., Xi, T., Liu, Y., Ai, F. \& Wu, Q., 2002. Thermal conductivity enhancement of suspensions containing nanosized alumina particles. Journal of applied physics, 91(7), pp.4568-4572.

Zheng, D., Wang, J., Chen, Z., Baleta, J. \& Sundén, B., 2020. Performance analysis of a plate heat exchanger using various nanofluids. International Journal of Heat and Mass Transfer, 158, p.119993. 